\title{
A Blind Watermarking Algorithm Research Based on Fast DCT
}

\author{
Siyu Lai \\ Department of Medical Imaging \\ North Sichuan Medical College \\ NanChong, China \\ 1sy 791211@126.com
}

\author{
Juan Wang* \\ College of Computer Science \\ China West Normal University \\ NanChong, China \\ *the corresponding author \\ wjuan0712@126.com
}

\begin{abstract}
This article exploits a fast algorithm to watermark implant and extract, which first transform $N \times N\left(N=2^{n}\right)$ two dimension DCT into one dimension DCT with $\mathrm{N}$ numbers of points. And then the adopted algorithm implants watermark using matrix decomposition and masterly adjusting interrelation of DCT coefficients, thus to make the visual distortion to be minima after a watermark being implanted in the image, in addition, this method is a blind approach. The results indicate that the algorithm enjoy high image reducing property and robustness to several common attacks when ensuring invisibility of watermark. Finally, it is also superior to R-C, index-mapping like matrix cut algorithms and can also improve calculating speed, which make it possible to apply for image protection.
\end{abstract}

Keywords-fast DCT; 2D-DCT; copyright protection; DCT modulus

\section{INTRODUCTION}

With the rapid development of multimedia and network, multimedia products (digital images, digital videos, digital audios, etc.) have been widely applied. As the multimedia digital products with the high performance of quality, effective storage, modification, transmission via computer network and especial precise duplication [1], it is difficult to prevent the action of illegal copy and falsify direct to digital products. Therefore, copyright protection became an instant issue to be address. Being an effective means of copyright protection, digital watermarking technology [2] attracted great attention both at home and abroad in recent years.

Digital Watermarking technology can generally be classified into two categories, spatial and transform domain watermarking [3]. Spatial domain methods directly modify pixels and the relevant algorithms are characterized by simplicity, low computational complexity and poor robustness. Transform domain approaches transform images in varies ways (DCT, DWT, DFT) and embed watermark. Comparatively, the latter have following advantages, that is, (1) watermark energy embedded in transform domain may distribute to all pixels in spatial domain and is conducive to robustness promotion; (2) can be easily combined with HVS and achieve the invisibility of watermark; (3) transform domain algorithms were compatible with most international images and videos compression standard and can directly implement watermark embedding in compressed domain. As a result, transform domain watermarking algorithms are the mainstream in these fields.

\section{ALGORITHM THEORY}

It is common knowledge that 2D-DCT [4] is a separable transform, thus, use the 1D-DCT fast algorithm which transform each dimension respectively to achieve 2DDCT, and this approach is known as determinant method that has been extensively adopted because of its simple structure. However, as it did not take relationship of two dimensions in 2D case into account and required a large amount of computation correspondingly. In actual image processing, the block transform methods are generally employed and the small size DCT (such as $4 \times 4,8 \times 8$ ) are mostly needed while the partition part occupying the majority of the processing time in actual system. For example, the time elapsed for DCT transform account for more than $1 / 3$ of the system time by using determinant method in a DCT-based image coding and decoding system. So we take a high effective algorithm of $4 \times 4$ DCT to dramatically shorten the execution time (about 1/5 of determinant method) and using the direct decomposition algorithm of 2D-DCT simultaneously. And it will be possible to design an effective fast DCT algorithm of arbitrary rectangular size by taking 4 $\times 4$ DCT [5] as the kernel.

We represent 2D image as $\{x(n, m) \mathrm{n}=0,1, \ldots, N-1, m=$ $0,1, \ldots M-1\}$

$$
\begin{aligned}
X(k, l)= & \sum_{n=0}^{N-1} \sum_{m=0}^{M-1} x(n, m) \cdot \cos \frac{\pi(2 n+1) k}{2 N} \cdot \cos \frac{\pi(2 m+1) l}{2 M} \\
& k=0,1 \cdots, N-1 ; l=0,1 \cdots, M-1
\end{aligned}
$$

It can also be expressed as the matrix form:

$$
X=C(N) \cdot x \cdot C^{T}(M)
$$

where $x$ is input data matrix $(N \times M), X$ is DCT coefficient matrix $(N \times M), C(N)$ is DCT transform matrix and $C^{T}(N)$ is transpose.

We change the order of input series in order to transform $2 \mathrm{D}$ to $1 \mathrm{D}$

$$
\bar{x}(i, j)= \begin{cases}x(2 i, 2 j) & 0 \leq i, j \leq \frac{N}{2}-1 \\ x(2 i, 2 N-2 j-1) & 0 \leq i \leq \frac{N}{2}-1, \frac{N}{2} \leq j \leq N-1 \\ x(2 N-2 i-1,2 j) & \frac{N}{2} \leq i \leq N-1,0 \leq j \leq \frac{N}{2}-1 \\ x(2 N-2 i-1,2 N-2 j-1) & \frac{N}{2} \leq i, j \leq N-1\end{cases}
$$

On circumstance of $4 \times 4$ DCT, 2D-DCT is transformed into the following style: 


$$
\begin{aligned}
& X(m, n)=\sum_{i=0}^{N-1} \sum_{j=0}^{M-1} \bar{x}(i, j) \cdot \cos \frac{m(4 i+1) \pi}{2 N} \cdot \cos \frac{n(4 j+1) \pi}{2 N} \\
& m, n=0,1, \cdots, N-1
\end{aligned}
$$

And the trigonometric function has the property of

$$
\begin{aligned}
& \cos \frac{m(4 i+1) \pi}{2 N} \cdot \cos \frac{n(4 j+1) \pi}{2 N}= \\
& \frac{1}{2}\left\{\cos \frac{m(4 i+1)+n(4 j+1)}{2 N}+\cos \frac{m(4 i+1)-n(4 j+1) \pi}{2 N}\right\}
\end{aligned}
$$

so formula (4) could be expressed as:

$$
X(m, n)=\frac{1}{2} \sum_{i=0}^{N-1} \sum_{j=0}^{N-1} \bar{x}(i, j) \cdot\left\{\begin{array}{l}
\cos \frac{m(4 i+1)+n(4 j+1)}{2 N} \pi+ \\
\cos \frac{m(4 i+1)-n(4 j+1)}{2 N} \pi
\end{array}\right\}
$$

define

$$
\begin{aligned}
& A(m, n)=\sum_{i=0}^{N-1} \sum_{j=0}^{N-1} \bar{x}(i, j) \cdot \cos \frac{m(4 i+1)+n(4 j+1)}{2 N} \pi \\
& B(m, n)=\sum_{i=0}^{N-1} \sum_{j=0}^{N-1} \bar{x}(i, j) \cdot \cos \frac{m(4 i+1)-n(4 j+1)}{2 N} \pi
\end{aligned}
$$

Then we merge the 2D-DCT with the above mentioned two new series.

$$
\begin{aligned}
& X(m, n)=\frac{1}{2}[A(m, n)+B(m, n)] \\
& m, n=0,1, \cdots, N-1
\end{aligned}
$$

Items of $\sum$ operation in (6) are formally the products of input data multiply cosine function and are similar to 1DDCT which could be transformed into 1D-DCT style by taking appropriate subscript mapping.

\section{IMBEDDING AND EXTRACTION OF WATERMARK}

\section{A. Watermark Built-in}

Given that the size of watermark image $W$ is $M 1 \times M 2$ and the size of original image $\mathrm{X}$ is $\mathrm{N} 1 \times \mathrm{N} 2$, then the embedding plan as follows [6]:

(1) Divide $X$ into $8 \times 8$ non-overlapped sub blocks, perform fast DCT transform on each small block and quantify them according to the JPEG quantization table.

(2) Select the appropriate embedded coefficient that described above.

(3) Transform binary image watermark into onedimensional sequence using dimension reduction. Load the watermark image first and get the two-dimensional matrix $W$, $W=\{W(i, j), 1 \leq i \leq m, 1 \leq j \leq n, W(i, j) \in\{0,1\}\}$, the size of $W$ is $m \times n$ and convert it to one-dimensional matrix $W_{t}, W_{t}=\left\{W_{t}\right.$ (i) $, 1 \leq i \leq m \times n, W(i, j) \in\{0,1\}\}$. In order to strengthen the stability and security of the watermarking system, perform bit extension on $W_{t}$ and the spreading factor is $\mathrm{cr}$. In image and video encoding system, DCT coefficients are commonly scanned follow the zigzag route as it shows in Figurel. Scanning ceased when detecting several coefficients after a certain one is all less than a threshold and marks all subsequent DCT coefficients as zero. Plus pseudo-random ordering to resist the trim operation and the ordering is controlled by keys to ensure the safe [7].

(4) Transform the revised coefficients using IDCT method, and then reconstruct the block to generate a watermark embedded image.

\section{B. Watermark Extraction}

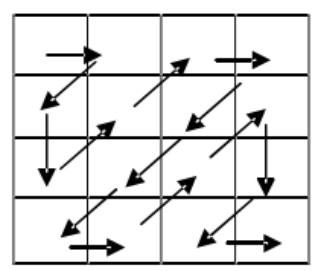

\begin{tabular}{|c|c|c|c|}
\hline 1 & 2 & 6 & 7 \\
\hline 3 & 5 & 8 & 13 \\
\hline 4 & 9 & 12 & 14 \\
\hline 10 & 11 & 15 & 16 \\
\hline
\end{tabular}

Figure 1. Zigzag scan style of DCT coefficient

We need first specify the embedded watermark coefficient when doing extraction job, and then calculate the value of embedded watermark-bit judging from the relationship between the first coefficient and the others by value. But the image might be distorted being under some sort of attack, which cause the first coefficient is no longer a max or min value. Thus the extraction algorithm in this article compare the first coefficient with the mean of max, min and average values of the rest coefficients and if the first coefficient is greater than the mean, then regard the extracted watermark bit is " 1 " or else " 0 ". The formula of extraction algorithm is denoted as follows [8]:

$$
W(i)= \begin{cases}1, i f \sum_{r=1}^{n u m} W_{t}((r-1) \times m \times n \times c r+i) \geq 0 \\ 0, \text { otherwise } \quad i=1,2, \cdots, m \times n \times c r\end{cases}
$$

expand $W(i), \mathrm{cr}$ is spreading factor and we get binary sequences $W_{p}(k)$ that containing $\{0,1\}$.

$$
W_{p}(k)=\left\{\begin{array}{l}
1, \text { if } \sum_{j=(k-1) \times c r+1}^{k \times c r} W(j) \geq 0 \\
0, \text { otherwise } \quad k=1,2, \cdots, m \times n
\end{array}\right.
$$

Mapping $W_{p}(k)$ and get two dimensional matrix $W^{\prime}, W^{\prime}=$ $\left\{W^{\prime}(i, j), 1 \leq i \leq m, 1 \leq j \leq n, W^{\prime}(i, j) \in\{0,1\}\right\}$ and finally we get the extracted watermark image.

\section{EXPERIMENTAL RESUlts AND PERFORMANCE ANALYSIS}

The experiment was developed under platform of $\mathrm{P} 4$ 2.8GHz, GeForce 7300GT, 1GB DDR2 and $\mathrm{VC}++6.0$. Original template was a 256 levels, $512 \times 512$ gray image Lena(Figure2.a) and the watermark template was a $164 \times$ 255 "China West Normal University" binary image as shown in figure2.b. Figure2.c was a visible watermark embedded image by using ordinary approach and Figure2.d was seemly an original alike image not containing any visible watermark. But in fact, Figure 2.d did containing an invisible watermark which was embedded take our adopted method on its lower right corner and these two images can be differentiated simply through check their file attributes about the size. And Figure2.f was the extracted watermark extracted from figure 
2.d without any disturbance and with Figure2.e was generated synchronously. According to the characteristics of the HVS (Human Visual System), the image fidelity rate was good except the background blur that was caused by stochastic noise, which enable the watermark enjoy a perfect invisible performance. It took us $98 \mathrm{~ms}$ to implement fast DCT transformation on Lena exploit our method while perform IDCT operation that cost $100 \mathrm{~ms}$. But if you apply a common DCT transformation it will consume inevitable a more long time. From this we can see that fast DCT algorithm enjoy great advantage of quick calculation speed and high efficiency.

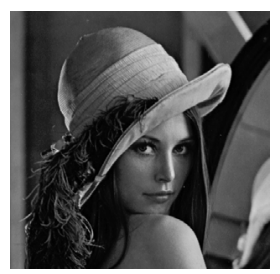

(a) original image

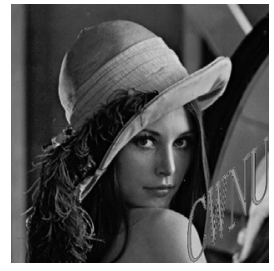

(c) visible watermark embedded image

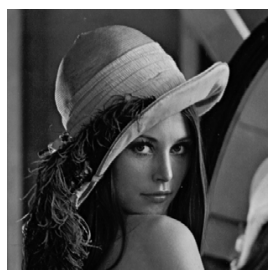

(e) image after watermark extraction

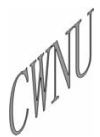

(b) original watermark

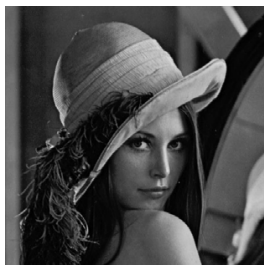

(d) invisible one

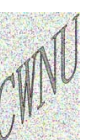

(f) extracted watermark

Figure 2. Process of watermarking implant and extract

TABLE I. COMPUTATIONAL COMPLEXITY AND AVERAGE

EXECUTION TIME OF $4 \times 4$ TRUNCATED DCT ALGORITHM

\begin{tabular}{|c|c|c|c|c|c|c|c|c|c|}
\hline \multirow{2}{*}{$\begin{array}{l}\text { Truncated } \\
\text { number }\end{array}$} & \multicolumn{3}{|c|}{ R-C } & \multicolumn{3}{|c|}{ Index-mapping } & \multicolumn{3}{|c|}{ This algorithm } \\
\hline & $M$ & $A$ & $T$ & $M$ & $A$ & $T$ & $M$ & $A$ & $T$ \\
\hline 1 & 0 & 15 & 0.17 & 0 & 15 & 0.14 & 0 & 14 & 0.06 \\
\hline 2 & 13 & 38 & 0.48 & 13 & 38 & 0.23 & 3 & 32 & 0.09 \\
\hline 3 & 17 & 43 & 0.58 & 16 & 43 & 0.24 & 6 & 41 & 0.11 \\
\hline 4 & 19 & 44 & 0.58 & 19 & 48 & 0.25 & 6 & 44 & 0.12 \\
\hline 5 & 22 & 49 & 0.66 & 19 & 53 & 0.27 & 7 & 53 & 0.13 \\
\hline 6 & 27 & 56 & 0.76 & 20 & 54 & 0.27 & 7 & 54 & 0.14 \\
\hline
\end{tabular}

addition and average execution time respectively (us)

In order to validate the robustness of algorithm in this paper, we conducted an aggressive experiment on the watermark embedded image take the following attack ways which were JPEG compression, shear and Gaussian filter, etc. Simulation results show that the embedded watermark was invisible and put up better robustness in dealing with the general image processing methods. Since we used a truncated algorithm to transform 2D matrix to 1D formation in the paper which reduced the computational complexity significantly, we designed a $4 \times 4$ DCT truncated algorithm merely calculate the first $1,2, \ldots, 6$ coefficients employ the $\mathrm{R}-\mathrm{C}$, index-mapping and this algorithm respectively in the light of the zigzag scanning style of DCT coefficient.

\section{CONCLUSIONS}

We adopted an improved fast DCT algorithm in this paper. The algorithm enjoy a comparatively low computation compared to others, furthermore the structure of this algorithm was simple and regular in view of signal flow process, thus this make it extremely suitable for software and hardware implementation. We simulated all the above mentioned algorithms under $\mathrm{VC}++6.0$ and took normalized factor and orthogonal factors into account. The experimental results show us this algorithm in possession of high efficiency comparatively just as above analyses figured out. And as DCT computation account for approximately $1 / 3$ of the entire time cost in a DCT based image and video encoding system. Thus, on one hand using this algorithm will significantly reduce encoding time which is avail to real time encoding. On the other hand, it would be possible to greatly enhance the rate of utilization of images and videos if solidify the algorithm in the form of hardware.

\section{REFERENCES}

[1] J. Sang, X. F. Liao, "The Concept and Approach for Digital Image Watermarking and Copyright Protection", Computer Science, vol. 32, Dec. 2005, pp. 111-115.

[2] Y. X. Yang, X. X. Niu. Theory and Applications of Digital Watermarking. BeiJin: Higher Education Press, 2006, pp.128-137.

[3] R. B. Wolfgang, C. I. Podilchuk and E. J. Delp. "Perceptual Watermarks for Digtal Image and Video", Proc. IEEE, Jan. 2008, pp. 1108-1126.

[4] R. X. Yin. A Fast DCT Algorithm and Filter Structure and Image Noise Reduction of Wavelet Transform Domain Research. South China University of Technology, 2000, pp. 15-40.

[5] R. X. Yin. "Fast Algorithm for Computing Prime-Length DCT Using Cyclic Convolutions", Journal of Data Acquisition \& Processing, vol. 34, Aug. 2001, pp. 42-46

[6] Q. M. Peng, X. F. Yang and S. Huan etc. "A Novel Adaptive Blind Digital Watermarking Algorithm”, Computer Science, vol. 32, Dec. 2005, pp. 63-66.

[7] M. Dorige, M. Vittorio and C. Alberto. "The Ant System: Optimization by a Colony of Cooperating Agents", IEEE Transactions on Systems, Man and Cybernetics, 1996, pp. 29-41.

[8] S. Huang, H. Q. Yang and W. Zhang etc. "A New Adaptive Watermarking Algorithm Based on DCT", Computer Science, vol 33, Jan. 2006, pp. 148-149. 